\title{
COMPACTIFYING A CONVERGENCE SPACE WITH FUNCTIONS
}

\author{
ROBERT P. ANDRÉ \\ Luther College \\ University of Regina Campus \\ Regina, Saskatchewan \\ Canada \\ S4S 0A2 \\ e-mail: andrer@meena.uregina.ca
}

(Received November 22, 1994 and in revised form March 25,1995)

ABSTRACT. A convergence space is a set together with a convergence structure. In this paper we discuss a method of constructing compactifications on a class of convergence spaces by use of functions.

KEYWORDS AND PHRASES: Compactification, convergence space, pretopological, singular compactification, singular set of a function.

A.M.S. SUBJECT CLASSIFICATION CODE: 54D35, 54A20, 54B20.

\section{INTRODUCTION.}

The terms whose definitions are given here for the sake of completeness are discussed in many textbooks in topology. A set $\Delta$ is a directed set if there exists a relation $\leq$ on $\Delta$ such that 1$) \delta \leq \delta$ for all $\delta$ $\in \Delta, 2) \delta_{1} \leq \delta_{2}$ and $\delta_{2} \leq \delta_{3}$ implies that $\delta_{1} \leq \delta_{3}$ and 3) if $\delta_{1}$ and $\delta_{2}$ belong to $\Delta$ then there exists some element $\delta_{3}$ in $\Delta$ such that $\delta_{1} \leq \delta_{3}$ and $\delta_{2} \leq \delta_{3}$. A net in a set $X$ is a function $\mathrm{s}: \Delta \rightarrow \mathrm{X}$ from a directed set $\Delta$ into $X$. If $\lambda$ is in the domain $\Delta$ of the net $s: \Delta \rightarrow X$ we will denote $s(\lambda)$ by $s_{\lambda}$ and the net $s$ in $X$ by ; $s_{\lambda}$ : $\lambda \in \Delta$ \}. For a directed set $\Delta$ we will denote by $\mu \Delta$ the set $\{\delta \in \Delta: \delta \geq \mu\}$. If $\Sigma$ is a subset of the directed set $\Delta$ then $\Sigma$ is cofinal in $\Delta$ (or frequently in $\Delta$ ) if $\mu \Delta \cap \Sigma \neq \varnothing$ for any $\mu \in \Delta$. If $t: \Sigma \rightarrow X$ is a function from $\Sigma$ into $X$ then $t$ is a subnet of $s: \Delta \rightarrow X$ if for any $\mu \in \Delta$ there exists a $\delta \in \Sigma$ such that $t[\delta \Sigma] \subseteq$ $s[\mu \Delta]$. A universal net (or ultranet) is a net with no proper subnet. The following ideas are introduced in So [18]. A convergence structure on a set $\mathrm{X}$ is a class $C$ of ordered pairs $(s, \mathrm{x})$ where $\mathrm{s}$ is a net in $\mathrm{X}$ and $\mathrm{x}$ $\epsilon \mathrm{X}$ such that for any $(\mathrm{s}, \mathrm{x})$ in $C$ the ordered pair $(\mathrm{t}, \mathrm{x})$ also belongs to $C$ if $\mathrm{t}$ is a subnet of $\mathrm{s}$. A convergence space $(\mathrm{X}, C)$ is a set $\mathrm{X}$ on which we have defined a convergence structure $C$. If a convergence structure $C$ is defined on a set $\mathrm{X}$ we will usually abbreviate $(\mathrm{X}, C)$ by $\mathrm{X}$. Also the phrase $s$ converges to $x$ (denoted by $\mathrm{s} \rightarrow \mathrm{x}$ ) will mean $(\mathrm{s}, \mathrm{x}) \in C$. A convergence space $\mathrm{X}$ is compact if every net in $\mathrm{X}$ has a convergent subnet in $\mathrm{X}$ and, finally, $\mathrm{X}$ is Hausdorff if no net in $\mathrm{X}$ converges to two distinct points in $\mathrm{X}$.

Throughout this paper $X$ will denote a convergence space. If $E \subseteq X$ then $\operatorname{cl}_{X} E=E \cup\{x \in X:$ there is some net $s$ in $E$ such that $s \rightarrow x\}$. Note that this closure operator is not necessarily idempotent, i.e., $c_{X} E$ may be a proper subset of $\mathrm{cl}_{\mathrm{X}} \mathrm{Cl}_{\mathrm{X}} \mathrm{E}$. A subset $\mathrm{E}$ of $\mathrm{X}$ is dense in $\mathrm{X}$ if $\mathrm{cl}_{\mathrm{X}} \mathrm{E}=\mathrm{X}$. If $\mathrm{f}$ is a map from $\mathrm{X}$ into a 
convergence space $Y$ then we say that $f$ is continuous if $s \rightarrow x$ in $X$ implies that $f \circ s \rightarrow f(x)$. Furthermore, if $f$ is one-to-one, continuous, and onto $Y$ and if $f \leftarrow: Y \rightarrow X$ is continuous then $f$ is called a homeomorphism from $\mathrm{X}$ onto $\mathrm{Y}$. As for topological spaces a compactification $\mathrm{Y}$ of $\mathrm{X}$ is an ordered pair $(\mathrm{Y}, \mathrm{h})$ where $\mathrm{Y}$ is a compact convergence space and $\mathrm{h}$ is a homeomorphism of $\mathrm{X}$ into $\mathrm{Y}$ such that $\mathrm{h}[\mathrm{X}]$ is dense in $\mathrm{Y}$. Given a compactification $\alpha \mathrm{X}$ of a space $\mathrm{X}$ the outgrowth (or remainder) of $\mathrm{X}$ in $\alpha \mathrm{X}$ is $\alpha \mathrm{XXX}$. Two compactifications $\alpha \mathrm{X}$ and $\gamma \mathrm{X}$ of $\mathrm{X}$ are said to be equivalent if there exists a homeomorphism between $\alpha \mathrm{X}$ and $\gamma \mathrm{X}$ that fixes the points of $\mathrm{X}$. We will say that $\mathrm{X}$ is pseudotopological at $x$ if $\mathrm{X}$ satisfies the following property: if every universal subnet of a net $\mathrm{s}$ in $\mathrm{X}$ converges to $\mathrm{x}$ then $\mathrm{s}$ converges to $\mathrm{x}$. We will sat that $X$ is pretopological at $x$ if $X$ satisfies the following property: If for a net of nets $S=\left\{s_{\delta}: \delta \in \Delta\right\}$ each net $\mathrm{s}_{\delta}=\left\{\mathrm{s}_{\delta}^{\mu}: \mu \in \Delta_{\delta}\right\}$ (where $\Delta_{\delta}$ is the domain of $\mathrm{s}_{\delta}$ ) converges to a point $\mathrm{x}_{\delta}$ in $\mathrm{X}$ and $\left\{\mathrm{x}_{\delta}: \delta \in \Delta\right\}$ converges to a point $\mathrm{x}$ in $\mathrm{X}$, then the net $\left\{\mathrm{s}_{\delta}^{\mu}: \delta \in \Delta, \mu \in \Delta_{\delta}\right\}$ ordered lexicographically by $\Delta$, then by $\Delta_{\delta}$, has a subnet which converges to $\mathrm{x}$ (i.e. $\mathrm{S}$ has a "diagonal net" that converges to $\mathrm{x}$ ). A convergence space $\mathrm{X}$ is said to be pseudotopological (pretopological) if $\mathrm{X}$ is pseudotopological (respectively pretopological) at every point in $X$. It is known that if a convergence space $X$ is both pseudotopological and pretopological and satisfies the property : "for a net $\mathrm{s}: \Delta \rightarrow \mathrm{X}, \mathrm{s}_{\delta}=\mathrm{x}$ for each $\delta \in \Delta$ implies $\mathrm{s}_{\delta} \rightarrow \mathrm{x}$ ", then we obtain a topology on $\mathrm{X}$ by defining the closure of a set $\mathrm{E}$ in $\mathrm{X}$ as $\operatorname{cl}_{\mathrm{X}} \mathrm{E}=\{\mathrm{x} \in \mathrm{X}:$ there is some net $\mathrm{s}$ in $\mathrm{E}$ such that $\dot{s} \rightarrow \mathrm{x}$ ) (see 1D of Willard [20]).

The following theorem is straightforward.

THEOREM 1. A convergence space $\mathrm{X}$ is compact if and only if every universal net in $\mathrm{X}$ converges.

We will say that a net $s=\left\{s_{\delta}: \delta \in \Delta\right\}$ in $X$ is eventually in $\mathrm{E} \subseteq \mathrm{X}$ if $\mathrm{s}[\mu \Delta] \subseteq \mathrm{E}$ for some $\mu \in \Delta$. The following lemma is Proposition 3.3 in Aarnes et al. [2].

LEMMA 2. If $s$ is a net in $X$, then $s$ is universal if and only if for each subset $E$ of $X, s$ is eventually in E or eventually in XVE.

In So [18] the author develops a method for constructing the one-point compactification of a noncompact Hausdorff convergence space $\mathrm{X}$ and discusses some of the properties of this compactification. In this paper we discuss a general method of constructing compactifications of a convergence space $\mathrm{X}$. In particular we use this method to construct a compactification to which every real-valued bounded function on $\mathrm{X}$ extends.

\section{PRELIMINARY DEFINITIONS AND RESULTS.}

The following technique for constructing compactifications is modeled on a method of constructing Hausdorff compactifications of locally compact Hausdorff spaces by using functions from $\mathrm{X}$ into a compact Hausdorff space K (see André [1], Chandler et al. [5], [6], Cain et al. [4], and Faulkner [11]). Let $\mathrm{f}: \mathrm{X} \rightarrow \mathrm{K}$ be a continuous function from the non-compact Hausdorff convergence space $\mathrm{X}$ into a compact Hausdorff topological space $K$. Let $Y=\operatorname{cl}_{K} f[X], K_{X}=\{F \subseteq X: F$ is compact $\}$ and $S(f)=$ $\cap\left\{\mathrm{cl}_{\mathrm{Y}} f[X \mathrm{XV}]: F \in K_{X}\right\}$. The subset $S(f)$ in $K$ will be called the singular set of $f$. Clearly $S(f)$ is closed and hence is compact in $\mathrm{Y}$.

LEMMA 3. Let $\mathrm{f}: \mathrm{X} \rightarrow \mathrm{K}$ be a function from a non-compact Hausdorff convergence space $\mathrm{X}$ into a compact Hausdorff topological space $\mathrm{K}$. If $\mathrm{s}: \Delta \rightarrow \mathrm{X}$ is a net in $\mathrm{X}$ that does not contain a convergent subnet then any subnet of the net fos in $\mathrm{Y}=\mathrm{cl}_{\mathrm{K}} \mathrm{f}[\mathrm{X}]$ converges to a point in $\mathrm{S}(\mathrm{f})$.

PROOF. Let $\mathrm{f}: \mathrm{X} \rightarrow \mathrm{K}$ be a function from a non-compact Hausdorff convergence space $\mathrm{X}$ into a compact Hausdorff topological space $\mathrm{K}$ and let $\mathrm{s}: \Delta \rightarrow \mathrm{X}$ be a net in $\mathrm{X}$ that does not contain a convergent subnet. Since $\mathrm{K}$ is compact the net fos has a convergent subnet $\mathrm{t}$ that converges to some point $\mathrm{y}$ in $\mathrm{Y}$. We 
claim that $y \in S(f)$. Let $F$ be a compact subset of $X$. Since $s$ has no convergent subnet in $X$ there exists a $\mu$ $\epsilon \Delta$ such that $s[\mu \Delta] \subseteq X V F$. Consequently fos $[\mu \Delta] \subseteq f[X V F]$. It follows that $y \in \operatorname{cl}_{Y}$ fos $[\mu \Delta] \subseteq \operatorname{cl}_{Y} f[X V F]$. Since $F$ was an arbitrary compact subset of $X, y \in \cap\left\{\operatorname{cl}_{K} f[X V]: F \in K_{X}\right\}=S(f)$ as claimed.

\section{THE MAIN RESULTS.}

Given an arbitrary continuous function $\mathrm{f}: \mathrm{X} \rightarrow \mathrm{K}$ from a non-compact Hausdorff convergence space $\mathrm{X}$ into a compact Hausdorff topological space $K$ let $X^{f}=X \cup S(f)$. We define a convergence structure on $X^{f}$ as follows. A net $s$ in $X^{f}$ converges to a point $x$ in $X$ if and only if $s$ is frequently in $X$ (i.e., $s$ has a cofinal subnet in $X)$ and $\left.s\right|_{X}$ converges to $x$. Let $f^{*}: X^{f} \rightarrow K$ be the function such that $\left.f^{*}\right|_{s(f)}$ is the identity function on $S(f)$ and $\left.f^{*}\right|_{X}=f$ on $X$. A net $s$ in $X^{f}$ converges to a point $y$ in $S(f)$ if and only if $s$ has no convergent subnet in $X$ and $f *_{o S}$ converges to $y$ in $S(f)$ (noting that, by lemma 3, y belongs to $S(f)$ ).

Let us now verify whether we have defined a convergence structure on $X^{f}$. We are required to show that if $s$ converges to $x$ in $X^{f}$ and $t$ is a subnet of $s$ then $t$ also converges to $x$. It will suffice to show this for a net $s$ in $X^{f}$ that converges to a point $x$ in $S(f)$. If $s$ is a net in $X^{f}$ that converges to a point $x$ in $S(f)$ then $s$ has no convergent subnet in $X$ and $f^{*}$ os converges to $x$ in $S(f)$. Let $t$ be a subnet of $s$. Then $f^{*}$ ot is a subnet of $\mathrm{f}^{*} \mathrm{~s}$ in $\mathrm{K}$ and so $\mathrm{f}^{*}$ ot converges to $\mathrm{x}$ in $\mathrm{K}$; hence $\mathrm{t}$ converges to $\mathrm{x}$. It follows that $\mathrm{Xf}^{\mathrm{f}}$ is a convergence space.

The following is a generalization of theorem 1.1 of Cain [4].

LEMMA 4. Let $\mathrm{f}$ be a continuous function from a Hausdorff convergence space $\mathrm{X}$ to a compact Hausdorff topological space $Z$. Let $Y=\operatorname{cl}_{Z} f[X]$ and $K_{X}=\{F \subseteq X: F$ is compact $\}$. Then the set $\{x \in K$ $: c_{X} f^{\leftarrow}[U]$ is not compact for any open neighbourhood $U$ of $x$ in $\left.K\right\}=S(f)\left(=\cap\left\{\operatorname{cl}_{Y} f[X V]: F \in K_{X}\right\}\right)$.

PROOF. Let $T=\left\{x \in K: \operatorname{cl}_{X} f^{\leftarrow}[U]\right.$ is not compact for any open neighbourhood $U$ of $x$ in $\left.K\right\}$. We will first show that $T \subseteq S(f)$. Let $F \in K_{X}$. Suppose $p$ belongs to $Y_{\text {cl }} f[X F]$. Then there exists an open neighbourhood $U$ of $p$ in $Y$ such that $f^{\leftarrow}[U] \subseteq F$ (since $Y$ is a compact Hausdorff topological space). Hence $p \notin T$ (since $c_{X} f^{\leftarrow}[U]$ is compact). We have thus shown that $T \subseteq c_{Y} f[X V]$. Since $F$ was arbitrarily chosen in $K_{X}$, it follows that $T \subseteq \cap\left(\operatorname{cl}_{Y} f[X V]: F \in K_{X}\right\}=S(f)$. Suppose now that $x$ belongs to $S(f)$. If $x$ belongs to $Y T$ then there exists an open neighbourhood $U$ of $x$ in $Y$ such that $c_{x} f^{\leftarrow}[U]$ is compact. But

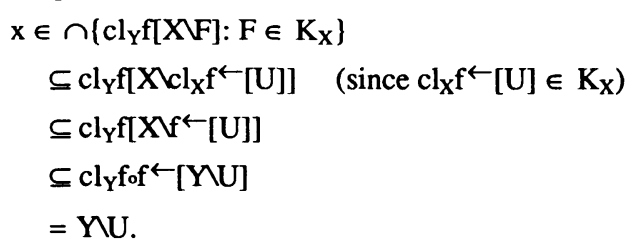

This contradicts that $x$ belongs to $U$. Consequently $\cap\left\{\operatorname{cl}_{Y} f[X F]: F \in K_{X}\right\} \subseteq T$. The lemma follows.

DEFINITION 5. We will say that a convergence space $\mathrm{X}$ is a $L C$ space if it satisfies the following property:

LC : Let $S=\left\{s_{\delta}: \delta \in \Delta\right\}$ be any net of nets in X such that each net $s_{\delta}=\left\{s_{\delta}^{\mu}: \mu \in \Delta_{\delta}\right\}$ (where $\Delta_{\delta}$ is the domain of $s_{\delta}$ ) in $S$ has no convergent subnet in $X$. Let $D=\left\{s_{\delta}^{\mu}: \delta \in \Delta, \mu \in \Delta_{\delta}\right\}$ ordered lexicographically by $\Delta$, then by $\Delta_{\delta}$. Then no subnet of $D$ is compact .

PROPOSITION 6. A Tychonoff topological space $X$ is locally compact if and only if $X$ is an LC space.

PROOF. Suppose $X$ is a locally compact Tychonoff space. We can then construct the Stone-Cech compactification $\beta X$ in which $X$ is open (see 18.4 of Willard [20]). Let $S=\left\{s_{\delta}: \delta \in \Delta\right\}$ be a net of nets in $X$ such that each net $s_{\delta}=\left\{s_{\delta}^{\mu}: \mu \in \Delta_{\delta}\right\}$ has no convergent subnet in $X$. Let $D=\left\{s_{\delta}^{\mu}: \delta \in \Delta, \mu \in \Delta_{\delta}\right\}$. 
Suppose that, for each $\delta \in \Delta, 1\left(t_{\delta}\right)$ is the limit of some convergent subnet $t_{\delta}=\left\{s_{\delta}^{\mu}: \mu \in \Sigma_{\delta}\right\}$ of $s_{\delta}$. Since $\beta \mathrm{X} \backslash \mathrm{X}$ is compact the net $\left\{1\left(\mathrm{t}_{\delta}\right): \delta \in \Delta\right\}$ has a subnet $\left\{1\left(\mathrm{t}_{\delta}\right): \delta \in \Sigma\right\}$ which converges to some point $\mathrm{x}$ in $\beta X X$. Let $T$ be any subnet of $\left\{s_{\delta}^{\mu}: \delta \in \Sigma, \mu \in \Sigma_{\delta}\right\}$ (itself a subnet of D). Then $T$ is of the form $T=\left\{s_{\delta}^{\mu}\right.$ : $\left.\delta \in \Lambda, \mu \in \Lambda_{\delta}\right\}$ (where $\left\{s_{\delta}: \delta \in \Lambda\right.$ \} is a subnet of $\left\{s_{\delta}: \delta \in \Sigma\right\}$ and $\left\{s_{\delta}^{\mu}: \mu \in \Lambda_{\delta}\right\}$ is a subnet of $\left\{s_{\delta}^{\mu}: \mu\right.$ $\left.\in \Sigma_{\delta}\right\}$ for each $\left.\delta \in \Sigma\right)$. It follows that $\left\{s_{\delta}^{\mu}: \mu \in \Lambda_{\delta}\right\}$ converges to $1\left(t_{\delta}\right)$, for each $\delta \in \Lambda$. Since $\beta X$ is topological it is pretopological. Hence the net $T=\left\{s_{\delta}^{\mu}: \delta \in \Lambda, \mu \in \Lambda_{\delta}\right\}$ has a subnet $H$ that converges to $x$ (since $\left\{l\left(t_{\delta}\right): \delta \in \Lambda\right\}$ converges to $x$ ). It then follows that every subnet of $H$ converges to $x$, i.e., no subnet of $H$ converges in $X$. This means that the subnet $T$ of $D$ has a subnet $H$ with no convergent subnet in $\mathrm{X}$. We have shown that $\mathrm{X}$ is a LC space.

We now prove the converse. Suppose $X$ is a Tychonoff $L C$ space that is not locally compact. Then the outgrowth $\beta X X$ of the Stone-Cech compactification $\beta X$ of $X$ is not closed in $\beta X$ (see 18.4 of [20]). Then there exists a net $s$ in $\beta X X$ that converges to a point $x$ in $X$. Let $D=\left\{s_{\delta}^{\mu}: \delta \in \Delta, \mu \in \Delta_{\delta}\right\}$, where $s_{\delta}$ and $s_{\delta}^{\mu}$ are as described in the previous paragraph. Since $\beta X$ is pretopological $D$ has a subnet $H$ that converges to $\mathrm{x}$. This means that $\mathrm{H}$ is compact, contradicting our hypothesis. Thus $\mathrm{X}$ must be locally compact.

We shall see that the LC property will guarantee that $X$ is dense in $X^{f}$.

We will now show that, for any continuous function $f: X \rightarrow K$ from a non-compact Hausdorff LC convergence space $\mathrm{X}$ into a compact Hausdorff topological space $\mathrm{K}, \mathrm{X}^{\mathrm{f}}$ is a Hausdorff compactification of $\mathrm{X}$.

THEOREM 7. If $\mathrm{f}: \mathrm{X} \rightarrow \mathrm{K}$ is a continuous function from a non-compact Hausdorff $L C$ convergence space $X$ into a compact Hausdorff topological space $K$ and $X^{f}=X \cup S(f)$ is equipped with the convergence structure described above, then $\mathrm{X}^{\mathrm{f}}$ is a compact, Hausdorff convergence space that densely contains X.

PROOF. We will begin by showing that $\mathrm{X}^{\mathrm{f}}$ is compact. Let $\mathrm{s}$ be a universal net in $\mathrm{X}^{\mathrm{f}}$ such that $\mathrm{s}$ is eventually in $\mathrm{X}$. Suppose $\mathrm{s}$ does not converge to a point in $\mathrm{X}$. Then the universal net $\mathrm{f}_{\mathrm{o}}$ s converges to some point $x$ in $S(f)$ (by lemma 3). Hence s converges to $x$ in $X^{f}$. Thus every universal net in $X$ converges in $\mathrm{X}^{\mathrm{f}}$. Obviously every universal net in $\mathrm{S}(\mathrm{f})$ converges in $\mathrm{X}^{\mathrm{f}}$. It follows that $\mathrm{X}^{\mathrm{f}}$ is compact.

To verify that $\mathrm{X}^{\mathrm{f}}$ is Hausdorff suppose $\mathrm{s}$ is a net in $\mathrm{X}^{\mathrm{f}}$ that converges to both $\mathrm{x}$ and $\mathrm{y}$ in $\mathrm{X}^{\mathrm{f}}$. If $\mathrm{x} \in \mathrm{X}$ then $\mathrm{s}$ is frequently in $\mathrm{X}$ and $\mathrm{s} \mathrm{X}_{\mathrm{X}}$ converges to $\mathrm{x}$. Since $\mathrm{s}$ has a convergent subnet in $\mathrm{X} s$ cannot converge to a point $y$ in $S(f)$; hence $y$ is in $X$. Since $X$ is Hausdorff, $x=y$. Suppose $\{x, y\} \subseteq S(f)$. This means that $s$ has no convergent subnet in $X$ and that $f *_{o s}$ converges to both $x$ and $y$ in $S(f)$; hence $x=y$ (since $S(f)$ is Hausdorff). Thus $\mathrm{X}^{\mathrm{f}}$ is Hausdorff.

We will now show that $X$ is dense in $X^{f}$. Let $x \in S(f)$ and let $U$ be an open neighbourhood of $x$ in $K$. We wish to show that there exists a net in $X$ that converges to $x$. Let $M$ be an open neighbourhood of $x$ in $K$ whose closure (in $K$ ) is contained in $U$. Then cl $\mathrm{X}^{\leftarrow}[\mathrm{M}]$ is non-compact (by lemma 4 ) and so $\mathrm{f}^{\leftarrow}[\mathrm{M}]$ contains a net $t$ with no convergent subnet in $X$. Since $\hat{r}^{*}$ ot is a net in $K, f^{*}$ ot has a convergent subnet that converges to some point $1(t)$ in $S(f)$ (by lemma 3 ). Hence $t$ has a subnet that converges to $1(t)$ (by definition of the convergence structure on $\left.X^{f}\right)$. Since $t \subseteq f^{\leftarrow}[M], f^{*}$ ot $\subseteq M$; hence $l(t) \in \operatorname{cl}_{K} f^{*}$ ot $\cap S(f) \subseteq c_{K} M \cap$ $S(f) \subseteq U \cap S(f)$. Hence for each open neighbourhood $U$ of $x$ in $K$ there exists a net $t$ with no convergent subnet in $X$ that converges to a point $l(t)$ in $U \cap S(f)$. It follows that there is a net $s=\left\{s_{\delta}: \delta \in \Delta\right\}$ of such nets in $\mathrm{X}$ whose limits $\mathrm{l}(\mathrm{s})=\left\{1\left(\mathrm{~s}_{\delta}\right): \delta \in \Delta\right\}$ in $\mathrm{S}(\mathrm{f})$ converges to $\mathrm{x}$. (The open neighbourhoods of a point $\mathrm{x}$ can be directed by defining $\mathrm{U} \leq \mathrm{U}_{1}$ and $\mathrm{U} \leq \mathrm{U}_{2}$ if $\mathrm{U} \subseteq \mathrm{U}_{1} \cap \mathrm{U}_{2}$ where $\mathrm{U}, \mathrm{U}_{1}$ and $\mathrm{U}_{2}$ are open 
neighbourhoods of $x$ ). For each $\delta \in \Delta$ let $\Delta_{\delta}$ denote the domain of $s_{\delta}$ and let $s_{\delta}=\left\{s_{\delta}^{\mu}: \mu \in \Delta_{\delta}\right\}$. We claim that the net $\mathrm{D}=\left\{\mathrm{s}_{\delta}^{\mu}: \delta \in \Delta, \mu \in \Delta_{\delta}\right\}$ ordered lexicographically by $\Delta$, then by $\Delta_{\delta}$, has a subnet that converges to $x$. Let $T$ be a subnet of $D$. Since $X$ was declared to be a LC space then $T$ has a subnet $H$ with no convergent subnet. We claim that $H$ converges to $x$. If $U$ is an arbitrary open neighbourhood of $x$ in $\mathrm{S}(\mathrm{f})$, then there exists an $\alpha \in \Delta$ such that $\left\{\mathrm{l}\left(\mathrm{s}_{\delta}\right): \delta \in \alpha \Delta\right\} \subseteq \mathrm{U}$. For $\delta \in \alpha \Delta,\left\{\mathrm{s}_{\delta}^{\mu}: \mu \in \Delta_{\delta}\right\}$ converges to $\mathrm{l}\left(\mathrm{s}_{\delta}\right)$; hence $\mathrm{f}{ }_{\mathrm{os}}{ }_{\delta}^{\mu}$ converges to $\mathrm{l}\left(\mathrm{s}_{\delta}\right)$. Hence for any $\delta \in \alpha \Delta$ there exists $\mu_{\alpha} \in \Delta_{\delta}$ such that $\left\{\mathrm{f}^{*_{\mathrm{os}}}{ }_{\delta}^{\mu}: \mu \in\right.$ $\left.\mu_{\alpha} \Delta_{\delta}\right\} \subseteq U$. Then, for any $\left.\delta \in \alpha \Delta, f^{\leftarrow}\left[\mathrm{f}^{*}{ }_{o}^{\mu}{ }_{\delta}^{\mu}: \mu \in \mu_{\alpha} \Delta_{\delta}\right\}\right] \subseteq \mathrm{f}^{\leftarrow}[\mathrm{U}]$ and so $\left[\mathrm{s}_{\delta}^{\mu}: \delta \in \alpha \Delta, \mu \in \mu_{\alpha} \Delta_{\delta}\right\}$ $\subseteq f \leftarrow[U]$. Hence $\mathrm{f}_{\circ} \mathrm{H}$ is eventually in $\mathrm{f} *_{\circ} f^{\leftarrow}[\mathrm{U}]=\mathrm{U}$. Since $\mathrm{U}$ was an arbitrary open neighbourhood of $\mathrm{x}$ $f * H$ converges to $x$. Since $H$ has no convergent subnet and $f^{*} \circ H$ converges to $x$ then $H$ converges to $x$ (by definition of the convergence structure on $\mathrm{X}^{f}$ ). This means that $\mathrm{x} \in \operatorname{cl}_{\mathrm{X}^{\mathrm{i}}} \mathrm{X}$ and so $\mathrm{X}$ is dense in $\mathrm{X}^{\mathrm{f}}$.

We have shown that $X^{f}$ is a Hausdorf compactification of $X$.

Observe that in the last part of the above proof we have shown that, if $\mathrm{X}$ is a non-compact Hausdorff LC convergence space and $\mathrm{f}$ is a continuous function from $\mathrm{X}$ into a compact Hausdorff topological space then $\mathrm{X}^{\mathrm{f}}$ is pretopological at each point $\mathrm{X}$ in $\mathrm{S}(\mathrm{f})$.

PROPOSITION 8. If $\mathrm{f}: \mathrm{X} \rightarrow \mathrm{K}$ is a function from a Hausdorff convergence space $\mathrm{X}$ into a compact Hausdorff topological space $K$ then the function $f$ extends continuously to a function $f^{*}: X^{f} \rightarrow K$ where $\left.\mathrm{f}^{*}\right|_{(\mathrm{f})}$ is the identity function on $\mathrm{S}(\mathrm{f})$.

PROOF. Clearly both $\left.\mathrm{f}^{*}\right|_{S(\mathrm{f})}$ and $\left.\mathrm{f} *\right|_{X}=f$ are continuous on $S(f)$ and $X$ respectively. Let $s$ be a net in $X$ that converges to $x$ in $S(f)$. Then $f^{*}$ os converges to $x=f^{*}(x)$ in $S(f)$ (by definition of the convergence structure on $\mathrm{X}^{\mathfrak{f}}$. Hence $\mathrm{f}^{*}$ os converges to $\mathrm{f}^{*}(\mathrm{x})$. Thus $\mathrm{f}^{*}$ is continuous on $\mathrm{X}^{\mathrm{f}}$.

EXAMPLE 9. Let $X$ be the real line. Let a net $s: \Delta \rightarrow X($ in $X)$ converge to a point $\mathbf{X}$ in $X$ if and only if $\mathrm{x}$ is an integer and for any $\alpha \in \Delta$ there exists a $\gamma \geq \alpha$ such that $s[\gamma \Delta] \subseteq(\mathrm{x}-1, \mathrm{x}]$. Observe that $X$ is a Hausdorff convergence space. To show that $X$ is a LC space let $S=\left\{s_{\delta}: \delta \in \Delta\right\}$ be a net of nets each of which has no convergent subnet in $X$. For each $\delta \in \Delta$, let $s_{\delta}=\left\{s_{\delta}^{\mu}: \mu \in \Delta_{\delta}\right\}$ and let $D=\left\{s_{\delta}^{\mu}: \delta \in \Delta, \mu \in\right.$ $\left.\Delta_{\delta}\right\}$ (ordered lexicographically). Let $\mathrm{T}=\left\{\mathrm{s}_{\delta}^{\mu}: \delta \in \Sigma, \mu \in \Sigma_{\delta}\right\}$ be a subnet of D. We claim that $\mathrm{T}$ is not compact (hence $X$ is a LC space). If $\delta \in \Sigma$ and $\mu \in \Sigma_{\delta}$ then there exists a $\gamma \in \Sigma_{\delta}$ such that $s_{\delta}^{\gamma}>s_{\delta}^{\mu}+1$ (since no cofinal subset of $s_{\delta}\left[\Sigma_{\delta}\right]$ is bounded in the space of real numbers $\mathbf{R}$ ). Consequently for each $\delta \in$ $\Sigma$ the net $s_{\delta}=\left\{s_{\delta}^{\mu}: \mu \in \Sigma_{\delta}\right\}$ has a countably infinite subnet $t_{\delta}=\left\{s_{\delta}^{\mu}: \mu \in \Lambda_{\delta}\right\}$ with no bounded interval in $X$ containing more than finitely many points of $t_{\delta}$. Let $\alpha \in \Sigma$ and $\beta \in \Lambda_{\delta}$. Then there exists a $\delta_{1}>\alpha$ in $\Sigma$ and $\mu_{1}$ in $\Lambda_{\delta}$ such that $s_{\delta_{1}}^{\mu_{1}}>s_{\alpha}^{\beta}+1$. Consequently we can construct a cofinal subset $H$ of $T$ such that $H$ has no convergent subnet. It follows that $T$ is not compact; hence $X$ is a LC space.

Let $f: X \rightarrow[-1,1]$ be the function from $X$ into $[-1,1]$ (equipped with the usual interval topology) defined as $f(x)=\sin (n)$ if $x \in(n-1, n]$ where $n$ is an integer. If $t$ is a net in $X$ that converges to a point $y \in$ $(n-1, n]$ for some integer $n$ then $t$ is eventually in $(n-1, n]$; hence fot is eventually $\sin (n)=f(n)$. It then follows that $f$ is continuous on $X$. We claim that if $U$ is an open interval in $[-1,1]$ then there exist an infinite number of integers $r$ such that $\sin (r) \in U$. It would then follow that $\mathrm{cl}_{X} \mathrm{f}^{\leftarrow}[\mathrm{U}]$ is not compact in $X$ for any open neighbourhood $U$ in $[-1,1]$. Let $Z$ denote the set of all integers. If $n \in Z$ let $[n \pi]$ denote the largest integer less than $n \pi$. We will use the following fact: The set $\{n \pi-[n \pi]: n \in Z\}$ is dense (equivalently, uniformly distributed) in [0,1]. (This fact is proved in most books on number theory). Let $\varepsilon$ $>0$ and $m$ be any number. We claim that there exists an integer $r$ such that $\sin (r) \in(\sin (m)-\varepsilon, \sin (m)+$ $\varepsilon$ ). There exists a $\delta>0$ such that $\sin [(m-\delta, m+\delta)] \subseteq(\sin (m)-\varepsilon, \sin (m)+\varepsilon)$. Suppose $m \geq 0$ and let $k$ 
be an even integer larger than $m+1$. Since the set $\{n \pi-[n \pi]: n \in Z\}$ is dense in $[0,1]$ then the set $\{k(n \pi$ $-[n \pi]): n \in Z\}$ is dense in $[0, k]$. Then there exists an integer $t \in Z$ such that $k(t \pi-[t \pi]) \in(m, m+\delta)$ $\subseteq[0, k]$ and so $\sin (k t \pi-k[t \pi]) \in(\sin (m)-\varepsilon, \sin (m)+\varepsilon)$. But $\sin (k t \pi-k[t \pi])=\sin (k t \pi) \cos (-k[t \pi])+$ $\sin (-k[t \pi]) \cos (k t \pi)=0+\sin (-k[t \pi])$, the sine of an integer. Thus if $r=-k[t \pi], \sin (r) \in(\sin (m)-$ $\varepsilon, \sin (m)+\varepsilon)$. It easily follows that $\sin ^{\leftarrow}[(\sin (m)-\varepsilon, \sin (m)+\varepsilon)] \cap \mathbf{Z}$ is infinite. We arrive at the same conclusion if we choose $m<0$. Hence $c_{X} f^{\leftarrow}[(\sin (m)-\varepsilon, \sin (m)+\varepsilon)]$ is non-compact in $X$. Thus $f[X]=$ $\sin [Z]$ is dense in $S(f)$. Hence $X^{f}$ is a compactification of $X$ whose outgrowth is $S(f)=[-1,1]$.

The example above illustrates a special type of compactification called a singular compactification. We define this below.

If the function $\mathrm{f}: \mathrm{X} \rightarrow \mathrm{K}$ from a Hausdorff convergence space $\mathrm{X}$ into a compact Hausdorff topological space $\mathrm{K}$ maps $\mathrm{X}$ into $\mathrm{S}(\mathrm{f})$ then we will say that $\mathrm{f}$ is a singular function and call $\mathrm{X}^{\mathrm{f}}$ a singular compactification of X. Singular compactifications of locally compact Hausdorff spaces are discussed extensively in André [1] and Chandler [5]. They are characterized as being those compactifications $\alpha \mathrm{X}$ of $\mathrm{X}$ whose outgrowth $\alpha \mathrm{X} \mathrm{X}$ is a retract of $\alpha \mathrm{X}$.

The following theorem follows easily from Proposition 8.

THEOREM 10. If $\mathrm{f}: \mathrm{X} \rightarrow \mathrm{K}$ is a singular function from a Hausdorff convergence space $\mathrm{X}$ into a compact Hausdorff topological space $K$ then $S(f)$ is a retract of $X^{f}$ under the function $f^{*}: X^{f} \rightarrow S(f)$ where $\left.f^{*}\right|_{X}=f$ and $\left.f *\right|_{S(f)}$ is the identity function on $S(f)$.

In example 9 above, the closed interval $[-1,1]=S(f)$ is a retract of $X^{f}$.

Proposition 11 is a generalization of lemma 1 in Chandler [5].

PROPOSITION 11. Let $\alpha \mathrm{X}$ be a Hausdorff compactification of a convergence space $\mathrm{X}$ such that $\alpha \mathrm{XVX}$ is compact. If $\mathrm{f}: \mathrm{X} \rightarrow \mathrm{K}$ is a continuous function from $\mathrm{X}$ into a compact Hausdorff topological space $K$ that extends to $f^{\alpha}: \alpha X \rightarrow K$ then $f^{\alpha}[\alpha X X]=S(f)$.

PROOF. Let $Y=\operatorname{cl}_{K} f[X]$. We are required to show that $f^{\alpha}[\alpha X X]$ is contained in $c_{Y} f[X V]$ for all $F \in$ $K_{X}$. Let $F \in K_{X}$ (where $K_{X}$ is as described above). Then $\alpha X X \subseteq \operatorname{cl}_{\alpha X}(X V)$ (since every net in $F$ has a convergent subnet in $F$ and $\alpha X X \subseteq \operatorname{cl}_{\alpha X}(F \cup X V F)=\operatorname{cl}_{\alpha X} F \cup \operatorname{cl}_{\alpha X} X V F=F \cup \operatorname{cl}_{\alpha X}(X V F)$ ). Hence $f^{\alpha}[\alpha X X] \subseteq f^{\alpha}\left[c_{\alpha X}(X V F)\right] \subseteq \operatorname{cl}_{Y} f[X V]$. Since this is true for all $F \in K_{X}, f^{\alpha}[\alpha X X] \subseteq \cap\left(\operatorname{cl}_{Y} f[X F]: F \in\right.$ $\left.K_{X}\right\}=S(f)$.

Let $p \in K f^{\alpha}[\alpha X X]$. Let $U$ be an open neighbourhood (in $K$ ) of $p$ such that $c_{K} U$ misses $f^{\alpha}[\alpha X X]$. Then $\operatorname{cl}_{\alpha X} f^{\alpha \leftarrow}[U] \subseteq f^{\alpha \leftarrow}\left[\operatorname{cl}_{Y} U\right] \subseteq X$. Hence $\operatorname{cl}_{X} f^{\leftarrow}[U]\left(=\operatorname{cl}_{\alpha X} f^{\alpha \leftarrow}[U]\right)$ is a compact subset of $X$. This implies that $p$ cannot belong to $S(f)$ (by lemma 4). Hence $S(f)=f^{\alpha}[\alpha X X]$.

LEMMA 12. Let $f: X \rightarrow K$ be a continuous function from a Hausdorff $L C$ convergence space $X$ into a compact Hausdorff topological space $\mathrm{K}$. If $\alpha \mathrm{X}$ is a Hausdorff compactification of $\mathrm{X}$ such that $\alpha \mathrm{XX}$ is compact and $\mathrm{f}$ extends continuously to $\mathrm{f}^{\alpha}: \alpha \mathrm{X} \rightarrow \mathrm{K}$ so that $\mathrm{f}^{\alpha}$ separates the points of $\alpha \mathrm{XX}$, then $\alpha \mathrm{X}$ is equivalent (as a compactification of $X$ ) to $X^{f}=X \cup S(f)$.

PROOF. By 11, $f^{\alpha}[\alpha X X]=S(f)$. We define a function $j: \alpha X \rightarrow X \cup S(f)$ as follows: $j(x)=f^{\alpha}(x)$ if $x$ belongs to $\alpha X X$ and $j(x)=x$ if $x$ belongs to $X$. Clearly $j$ is one-to-one. We now verify that $j$ is continuous. Let $\mathrm{s}: \Delta \rightarrow \mathrm{X}$ be a net in $\mathrm{X}$ such that $\mathrm{s}$ converges to $\mathrm{x}$ in $\alpha \mathrm{XXX}$. We wish to show that jos $\rightarrow$ $j(x)\left(=f^{\alpha}(x)\right)$ in $X^{f}$. Equivalently we wish to show that $s \rightarrow f^{\alpha}(x)$ in $X^{f}$. Suppose $s \rightarrow y$ in $X^{f}$. If $y \neq f^{\alpha}(x)$ then there exists an open neighbourhood $U$ of $y$ in $K$ such that $f^{\alpha}(x) \in K \operatorname{Kcl}_{K} U$. By 8 the function $f: X \rightarrow$ $K$ extends continuously to a function $\mathrm{f}^{*}: \mathrm{X}^{\mathrm{f}} \rightarrow \mathrm{K}$ such that $\left.\mathrm{f}^{*}\right|_{S(f)}$ is the identity function on $\mathrm{S}(\mathrm{f})$. Then $\mathrm{f}_{\circ} \mathrm{os} \rightarrow \mathrm{f}^{*}(\mathrm{y})=\mathrm{y} \in \mathrm{U}$, and so there exists a $\mu \in \Delta$ such that $\mathrm{f}^{*} \mathrm{os}[\mu \Delta] \subseteq U$. It follows that $s[\mu \Delta] \subseteq$ 
$\mathrm{f}^{* \leftarrow}[\mathrm{U}]$. Similarly, since $\mathrm{f}^{\alpha}$ is continuous on $\alpha \mathrm{X}, \mathrm{f}^{\alpha}{ }_{\mathrm{os}} \rightarrow \mathrm{f}^{\alpha}(\mathrm{x})$; hence there exist a $\delta \in \Delta$ such that $\mathrm{f}^{\alpha_{o S}}[\delta \Delta] \subseteq \mathrm{Klcl}_{\mathrm{K}} \mathrm{U}$ and $\mathrm{s}[\delta \Delta] \subseteq \mathrm{f}^{\alpha \leftarrow}\left[\mathrm{K} \operatorname{lcl}_{\mathrm{K}} U\right]$. This implies that $\mathrm{f}^{\leftarrow}\left[\mathrm{K} / \mathrm{Kl}_{\mathrm{K}} \mathrm{U}\right] \cap \mathrm{f}^{\leftarrow}\left[\mathrm{cl}_{\mathrm{K}} \mathrm{U}\right]$ cannot be empty, a contradiction. Hence $y=f^{\alpha}(x)$. Since $s \rightarrow y, s \rightarrow f^{\alpha}(x)$ as required. Thus $j$ is a continuous function.

We now proceed similarly to show that $\mathrm{j} \leftarrow$ is continuous. Let $\mathrm{s}: \Delta \rightarrow \mathrm{X}$ be a net in $\mathrm{X}$ that converges to $x \in S(f)$. We wish to show that $j^{\leftarrow}$ os $\rightarrow j^{\leftarrow}(x)=f^{\alpha \leftarrow}(x)$. Equivalently we wish to show that $s \rightarrow f^{\alpha \leftarrow}(x)$. Suppose $s \rightarrow y$ in $\alpha X X$. We claim that $y=f^{\alpha \leftarrow}(x)$. If $y \neq f^{\alpha \leftarrow}(x)$ then $f^{\alpha}(y) \neq f^{\alpha} f^{\alpha \leftarrow}(x)=x$ (since $f^{\alpha}$ is one-to-one on $\alpha \mathrm{XX})$. Hence there exists an open neighbourhood $U$ of $\mathrm{f}^{\alpha}(\mathrm{y})$ such that $\mathrm{x} \in \boldsymbol{\alpha X \backslash \mathrm { I } _ { \alpha X } U}$. Since $f^{\alpha}: \alpha X \rightarrow K$ is continuous $f^{\alpha}{ }_{o S} \rightarrow f^{\alpha}(y)$. Hence there exists a $\mu \in \Delta$ such that $f^{\alpha}{ }_{o s}[\mu \Delta] \subseteq U$; then $s[\mu \Delta] \subseteq f^{a \leftarrow}[U]$. Similarly, since $f^{*}: X^{f} \rightarrow K$ is continuous and $s \rightarrow x$ in $X^{f}, f^{*} o s \rightarrow f^{*}(x)=x$; hence there exists a $\delta \in \Delta$ such that $f^{*}{ }_{\mathrm{os}}[\delta \Delta] \subseteq \mathrm{Klcl}_{\mathrm{K}} \mathrm{U}$. Thus $\mathrm{s}[\delta \Delta] \subseteq \mathrm{f}^{* \leftarrow}\left[\mathrm{K} \backslash \mathrm{cl} \mathrm{K}_{\mathrm{K}} \mathrm{U}\right]$. It follows that $\mathrm{f}^{\leftarrow}\left[\mathrm{K} \mathrm{Cl}_{\mathrm{K}} \mathrm{U}\right]$ $\cap f^{\leftarrow}\left[\mathrm{cl}_{\mathrm{K}} \mathrm{U}\right]$ is non-empty, a contradiction. Hence $\mathrm{y}=\mathrm{f}^{\alpha \leftarrow}(\mathrm{x})$ as claimed. It then follows that $\mathrm{s} \rightarrow \mathrm{f}^{\mathrm{\alpha} \leftarrow}(\mathrm{x})$ and so $\mathrm{j} \leftarrow$ is continuous. Since $\mathrm{j}: \alpha \mathrm{X} \rightarrow \mathrm{X}^{\mathrm{f}}$ is a homeomorphism that fixes the points of $\mathrm{X}, \boldsymbol{\alpha X}$ and $\mathrm{X}^{\mathrm{f}}$ are equivalent compactifications of $\mathrm{X}$.

If $G$ is a collection of real-valued bounded functions on $\mathrm{X}$, the evaluation map $\mathrm{e}_{G}$ induced by $G$ is the function $\mathrm{e}_{G}: \mathrm{X} \rightarrow \Pi\left\{\mathrm{I}_{\mathrm{g}}: \mathrm{g} \in G\right\}$ (where, for each $\mathrm{g}, \mathrm{I}_{\mathrm{g}}$ is a closed interval containing $\mathrm{g}[\mathrm{X}]$ ) defined by $\mathrm{e}_{G}(\mathrm{x})=\langle\mathrm{g}(\mathrm{x})\rangle_{\mathrm{g} \in G}$. Note that the closure in $\Pi_{\mathrm{g} \in G} \mathrm{I}_{\mathrm{g}}$ of $\mathrm{e}_{G}[\mathrm{X}]$ is a compact set.

Let $\mathrm{X}$ be a Hausdorff LC convergence space and let $C^{*}(X)$ denote the collection of all real-valued bounded continuous functions on $\mathrm{X}$. We will show that, by using the above method of constructing compactifications of a Hausdorff $\mathrm{LC}$ convergence space we may construct a compactification $\mathrm{X}^{*}$ of $\mathrm{X}$ in which $\mathrm{X}$ is $C^{*}$-embedded, i.e., a compactification $\mathrm{X}^{*}$ of $\mathrm{X}$ where every function $\mathrm{f}$ in $\mathrm{C}^{*}(\mathrm{X})$ extends continuously to a real-valued function $\mathrm{f}^{*}$ on $\mathrm{X}^{*}$. Consider the evaluation map $\mathrm{e}_{\mathrm{C}^{*}(\mathrm{X})}$ induced by $\mathrm{C}^{*}(X)$ from $X$ into $\Pi\left\{I_{g}: g \in C^{*}(X)\right\}$ (where, for each $g, I_{g}$ is a closed bounded interval containing $g[X]$ ). Then $X^{e_{C^{*}}(X)}=X \cup S\left(e_{C^{*}(X)}\right)$. Since $X$ is a LC space and $e_{C^{*}(X)}$ maps $X$ into a compact Hausdorff topological space, $X^{e^{*}(X)}$ is a Hausdorff compactification of $X$. Now $e_{C^{*}(X)}$ extends continuously to $e_{C^{*}}(X)^{*}$ on $X^{\mathrm{e}^{*}(\mathrm{X})}$ where $\mathrm{e}_{\mathrm{C}^{*}(\mathrm{X})}{ }^{*}$ restricted to $S\left(\mathrm{e}_{\mathrm{C}^{*}(\mathrm{X})}\right)$ is the identity function. If $\mathrm{f} \in \mathrm{C}^{*}(X)$ and $\pi_{\mathrm{f}}: \Pi\left\{\mathrm{I}_{\mathrm{g}}: \mathrm{g} \in\right.$ $\left.\mathrm{C}^{*}(\mathrm{X})\right\} \rightarrow \mathrm{I}_{\mathrm{f}}$ where $\pi_{\mathrm{f}^{\circ} \mathrm{C}^{*}(\mathrm{X})}(\mathrm{x})=\mathrm{f}(\mathrm{x})$ then the map $\mathrm{f}^{*}=\pi_{\mathrm{f}^{*}} \mathrm{C}^{*}(\mathrm{X})^{*}$ is a continuous extension of $\mathrm{f}$ to $X^{e^{*}(X)}$ mapping a point $x$ in $S\left(e_{C^{*}(X)}\right)$ to $f^{*}(x)$ in $I_{f}$. We have just constructed a compactification of $X$ in which $\mathrm{X}$ is $\mathrm{C}^{*}$-embedded and whose outgrowth is a compact Hausdorff topological space. We will denote

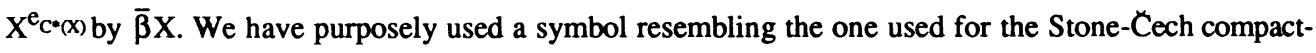
ification $\beta X$ of a locally compact Hausdorff topological space $X$ since the method used to construct $\bar{\beta} X$ mimics one used to construct $\beta X$ (see 2.2 of André [1]).

The family of all Hausdorff compactifications of a Hausdorff convergence space can be partially ordered as follows: $\alpha \mathrm{X} \leq \gamma \mathrm{X}$ if there exists a continuous function $\mathrm{h}: \gamma \mathrm{X} \rightarrow \alpha \mathrm{X}$ from $\gamma \mathrm{X}$ onto $\alpha \mathrm{X}$ such $\left.\mathrm{h}\right|_{\mathrm{X}}$ fixes the points of $\mathrm{X}$.

THEOREM 13. Let $X$ be a Hausdorff LC convergence space. Then $\bar{\beta} X \geq \alpha X$ for all Hausdorff compactifications $\alpha \mathrm{X}$ of $\mathrm{X}$ whose outgrowth $\alpha \mathrm{X} \backslash \mathrm{X}$ is a compact Hausdorff topological space that is $\mathrm{C}^{*}$ embedded in $\alpha X$. Also $\bar{\beta} X \geq \gamma X$ for any compactification $\gamma X$ where $\gamma X$ is of the form $X^{f}=X \cup S(f)$ where $f: X \rightarrow K$ is a continuous function from $X$ into a compact Hausdorff topological space $K$.

PROOF. Let $\mathrm{X}$ be a non-compact Hausdorff LC convergence space. Let $\alpha \mathrm{X}$ be a Hausdorff compactification of $\mathrm{X}$ such that $\alpha \mathrm{XX}$ is a compact topological space that is $\mathrm{C}^{*}$-embedded in $\alpha \mathrm{X}$. We are required to show that $\alpha X \leq \bar{\beta} X$. Let $M=\left\{f \in C^{*}(\alpha X)\right.$ : $f$ is a continuous extension to $\alpha X$ of a function in 
$\left.C^{*}(\alpha X X)\right\}$. Since $C^{*}(\alpha X \backslash X)$ separates the points of $\alpha X X, M$ separates the points of $\alpha X X$. Hence, $e_{M}$ is one-to-one on $\alpha X X$. Let $T=\left.C^{*}(\alpha X)\right|_{X}$. Since each function in $T$ extends continuously to $\bar{\beta} X, e_{T}$ extends continuously to a function $\mathrm{e}_{\mathrm{T}}{ }^{\beta}$ on $\bar{\beta} \mathrm{X}$. Let $\pi_{\beta \alpha}: \beta \mathrm{X} \rightarrow \alpha \mathrm{X}$ be a function from $\beta \mathrm{X}$ to $\alpha \mathrm{X}$ which maps $\mathrm{e}_{\mathrm{T}}{ }^{\beta \leftarrow}(\mathrm{x}) \cap \beta \mathrm{X} \backslash \mathrm{X}$ to $\mathrm{e}_{\mathrm{T}}{ }^{\alpha \leftarrow}(\mathrm{x}) \cap \alpha \mathrm{X} \backslash \mathrm{X}$ for each $\mathrm{x} \in \mathrm{e}_{\mathrm{T}}{ }^{\alpha}[\alpha \mathrm{X} \backslash \mathrm{X}]$ and which fixes the points of $\mathrm{X}$ (noting that $\mathrm{e}_{\mathrm{T}}^{\beta}[\beta \mathrm{X}]=\mathrm{e}_{\mathrm{T}}^{\alpha}[\alpha \mathrm{X}]$ ). It is easily verified that $\pi_{\beta \alpha}$ is continuous. Hence $\alpha \mathrm{X} \leq \bar{\beta} \mathrm{X}$.

Suppose that $\gamma \mathrm{X}$ is a compactification of the form $\mathrm{X}^{f}=\mathrm{X} \cup \mathrm{S}(\mathrm{f})$ where $\mathrm{f}: \mathrm{X} \rightarrow \mathrm{K}$ is a continuous function from $X$ into a compact Hausdorff topological space $K$. Let $Y=\operatorname{cl}_{K} f[X]$. If $g \in C^{*}(Y)$ then gof* $\in$ $C^{*}\left(X^{f}\right)$. Since $C^{*}(Y)$ separates the points of $Y$ and $S(f) \subseteq Y$ then the family $\left\{g \circ f^{*}: g \in C^{*}(Y)\right\}$ separates the points of $S(f)$. Consequently if $T=C^{*}\left(X^{f}\right), e_{T}$ is one-to-one on $S(f)$. Let $M=\left.T\right|_{X}$. Then $e_{M}$ extends continuously to the function $\left(e_{M}\right)^{\beta}$ on $\bar{\beta} X$. Let $\pi_{\beta X^{f}}: \beta X \rightarrow X^{f}$ be a function from $\beta X$ onto $X^{f}$ which maps $\left(e_{M}\right)^{\beta \leftarrow}(x) \cap \bar{\beta} X X X$ to $e_{T}^{\leftarrow}(x) \cap S(f)$ for each $x \in\left(e_{M}\right)^{\beta}[\gamma X X]$ and which fixes the points of $X$. Again it is easily verified that $\pi_{\beta X^{f}}$ is continuous. Hence $\gamma \mathrm{X} \leq \bar{\beta} \mathrm{X}$.

EXAMPLE 14. Let $\omega_{1}$ denote the first uncountable ordinal. Let $X=\cup\left\{I_{i}: i \in\left[0, \omega_{1}\right)\right\}$ where, for each $i \in\left[0, \omega_{1}\right), I_{i}$ is the unit interval $[0,1]$. We will say that a net $s$ in $X$ converges to a rational number $X$ in $I_{i}$ if and only if $s$ is eventually in every open interval in $I_{i}$ that contains $x$. A net $s$ converges to an irrational number $x$ in $I_{i}$ if and only if $i$ has an immediate predecessor $i-1$ and $s$ is eventually in every open interval containing $x$ in $I_{i-1}$. Thus a net $s$ in $I_{i}$ will always converge in $I_{i} \cup I_{i+1}$. It is easily seen that $X$ is a noncompact Hausdorff convergence space. We will describe some other properties of $\mathrm{X}$.

We claim that $X$ is not pretopological. Let $S=\left\{s_{\delta}: \delta \in \Delta\right\}$ be a net of nets in $I_{i}\left(i \in\left[0, \omega_{1}\right)\right)$ where each net $s_{\delta}$ converges to some irrational number $\mathrm{l}\left(\mathrm{s}_{\delta}\right)$ in $\mathrm{I}_{\mathrm{i}+1}$. Suppose the nets are chosen so that the net $\left\{1\left(s_{\delta}\right): \delta \in \Delta\right\}$ converges to an irrational number $y$ in $I_{i+2}$. For each $\delta \in \Delta$, let $s_{\delta}=\left\{s_{\delta}^{\mu}: \mu \in \Delta_{\delta}\right\}$ and let $\mathrm{D}=\left\{\mathrm{s}_{\delta}^{\mu}: \delta \in \Delta, \mu \in \Delta_{\delta}\right\}$ (ordered lexicographically). Since $\mathrm{D} \subseteq \mathrm{I}_{\mathrm{i}}$ no subnet of $\mathrm{D}$ can converge to a point in $I_{i+2}$ (since all nets in $I_{i}$ converge in $I_{i} \cup I_{i+1}$ ). Hence no subnet of $D$ can converge to $y$. Thus $X$ is not pretopological.

Also observe that for the irrational number $\pi / 4$ in some $I_{i}$ the net $s=\left\{s_{\delta}: \delta \in \Delta\right\}$ where $s_{\delta}=\pi / 4$ for all $\delta \in \Delta$ converges to the irrational number $\pi / 4$ in $I_{i+1}$. Hence a constant net $s$ in $X$ where each member is the same number $r$ in $X$ need not necessarily converge to $r$.

We now claim that $X$ is a LC space. Let $S=\left\{s_{\delta}: \delta \in \Delta\right\}$ be a net of nets each of which has no convergent subnet in $X$. For each $\delta \in \Delta$, let $s_{\delta}=\left\{s_{\delta}^{\mu}: \mu \in \Delta_{\delta}\right\}$. If $\delta \in \Delta$, s $\delta$ has no convergent subnet in $X$ hence no cofinal subset of $s_{\delta}$ is contained in any $I_{i}$. Thus $s_{\delta}$ has a subnet $t_{\delta}=\left\{s_{\delta}^{\mu}: \mu \in \Sigma_{\delta}\right\}$ such that $t_{\delta} \cap I_{i}$ is finite for each $i \in\left[0, \omega_{1}\right)$. Let $D=\left\{s_{\delta}^{\mu}: \delta \in \Delta, \mu \in \Sigma_{\delta}\right\}$ (ordered lexicographically). Let $T=$ $\left\{\mathrm{s}_{\delta}^{\mu}: \delta \in \Lambda, \mu \in \Lambda_{\delta}\right\}$ (where $\Lambda$ and $\Lambda_{\delta}$ are cofinal in $\Delta$ and $\Sigma_{\delta}$ respectively). Let $\alpha \in \Lambda$ and $\beta \in \Lambda_{\delta}$. If $s_{\alpha}^{\beta} \in I_{i}$ then there exists a $\delta_{1}>\alpha$ in $\Lambda$ and $\mu_{1}$ in $\Lambda_{\delta}$ such that $s_{\delta_{1}}^{\mu_{1}} \in I_{i+1}$. Consequently we can construct a subnet $\mathrm{H}$ of $\mathrm{T}$ such that $\mathrm{H}$ has no convergent subnet. It follows that $\mathrm{T}$ is non-compact; hence $\mathrm{X}$ is a $\mathrm{LC}$ space.

Let $\mathrm{f}$ be any continuous function from $\mathrm{X}$ into a compact Hausdorff topological space $\mathrm{K}$ and let $\mathrm{u}$ be an irrational number in $[1,0]$. Let $U=\left\{u_{i}: i \in\left[0, \omega_{1}\right)\right\}$ where $u_{i}=u$ for all $i \in\left[0, \omega_{1}\right)$ and let $s=\left\{s_{\delta}: \delta \in\right.$ $\Delta$ \} be the constant net in some $I_{i}$ such that $s_{\delta}=u_{i}$ for all $\delta \in \Delta$. Then the net s converges to the number $u_{i}$ +1 . (Note that $U$ is non-compact). Since $f(s)$ is a constant net in $K$ and $f$ is continuous $f\left(u_{i+1}\right)=f[s]$. It follows easily that $f[U]$ must be a singleton set in $K\left\{f\left(u_{0}\right)\right\}$. Let $x$ be an arbitrary point in $f[X]$ and let $V$ be an open neighbourhood of $x$ in $\mathrm{cl}_{K} f[X]$. If $x$ is an irrational number than $\mathrm{cl}_{\mathbf{X}} \mathrm{f}^{\leftarrow}[\mathrm{V}]$ is non-compact (since clx $\mathrm{f}^{\leftarrow}[\mathrm{V}]$ contains a set such as $\mathrm{U}$ above). Suppose $\mathrm{x}$ is a rational number in some $\mathrm{I}_{\mathrm{i}}$. Let $\mathrm{s}=\left\{\mathrm{s}_{\delta}: \delta\right.$ 
$\in \Delta$ \} be a net of irrational numbers in $I_{1}$ such that $s$ converges to $x$. Since $f$ is continuous the net $f[s]$ converges to $f(x)$ in $\operatorname{cl}_{K} f[X]$. Hence there exists an $\alpha \in \Delta$ such that $f[s[\alpha \Delta]] \subseteq V$. This means that $V$ contains the image of an irrational number in $\mathrm{I}_{\mathrm{i}}$. Again it follows that $\mathrm{cl}_{\mathrm{X}} \mathrm{f}^{\leftarrow}[\mathrm{V}]$ is not compact. Then, by lemma $4, \mathrm{cl}_{\mathrm{K}} f[X]$ is the singular set $S(f)$ of $f$, i.e., $f$ is a singular function. Since $f$ is an arbitrary function every function from $\mathrm{X}$ into a compact Hausdorff topological space is singular. Hence the compactification $\bar{\beta} X=X^{e^{*}(X)}$ is a singular compactification (since $e_{C^{*}(X)}$ is singular).

\section{REFERENCES}

1. ANDRÉ, Robert P. On the supremum of singular compactifications, Submitted.

2. AARNES, J. F. and ANDENAES, P. R. On nets and filters, Math. Scand. 31 (1972), 285-292

3. CHANDLER, Richard E. Hausdorff Compactifications, Marcel Dekker, Inc., New York and Basel, 1976.

4. CAIN Jr., George L., CHANDLER, Richard E. and FAULKNER, Gary D. Singular sets and remainders, Trans. Amer. Math Soc. 268 (1981), 161-171.

5. CHANDLER, Richard E., FAULKNER, Gary D. Singular Compactifications: the order structure. Proceedings of the American Mathematical Society 100, number 2 (1987), 377-382.

6. CHANDLER, Richard E., FAULKNER, Gary D., GUGLIELMI, Josephine P., and Margaret MEMORY, Generalizing the Alexandroff-Urysohn double circumference construction, Proceedings of the American Mathematical Society 83 (1981), 606-609.

7. CATERINO, A., FAULKNER, G. D., VIPERA, M. C. Two Applications of Singular Sets to the Theory of Compactifications, To appear in Rendiconti Dell'Insitute di Mat. dell'Univ. di Thieste.

8. COMFORT, W. W. Retractions and other continuous maps from $\beta X$ onto $\beta X X$. Trans. Amer. Math. Soc. 114 (1965) 843-847.

9. ENGELKING, Ryszard General Topology, Polish Scientific publishers, Warszawa, Poland, 1977.

10. FAULKNER, Gary D. Minimal Compactifications and their associated function spaces, Proceedings of the American Mathematical Society 108, Number 2, February 1990, 541-546.

11. Compactifications from mappings, To appear in Atti del IV Convigno di Topologia, Rendic. Circ. Mat. Univ. Palermo

12. FROLIK, Z. Concerning topological convergence of sets, Czechoslovak Math. Vol. 10(85) (1960), 168-180.

13. GUGLIELMI, Josephine $\mathrm{P}$. Compactifications with singular remainders, $\mathrm{Ph} . \mathrm{D}$ thesis, North Carolina State University, 1986.

14. KELLEY, John L. General Topology, D. Van Nostrand Company inc., New York, 1955.

15. KENT, D. C.and RICHARDSON G. D. Compactifications on convergence spaces, International Journal of Mathematics and Mathematical Sciences, Vol.2(3) (1979) 345-368.

16. RICHARDSON, G. D. and KENT, D. C. The Star Compactifications, International Journal of Mathematics and Mathematical Sciences, Vol4(3) (1981), 451-472. 
17. PEARSON, B. J. Spaces defined by convergence classes on nets, Glasnik Matematicki, Vol.23(43) (1988), 135-142.

18. SO, Shing S. One-point compactification of convergence spaces, International Journal of Mathematics and Mathematical Sciences, Vol.17 No.2 (1994) 277-282.

19. WALKER, R. The Stone-Cech Compactification, Springer-Verlag, New York, 1974.

20. WILlARD, S. General Topology, Addison-Wesley Publishing Company, 1970. 


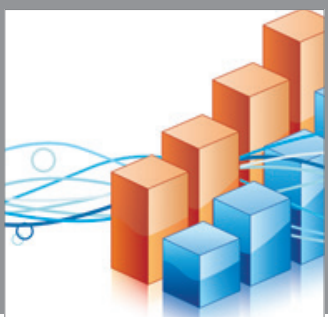

Advances in

Operations Research

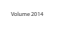

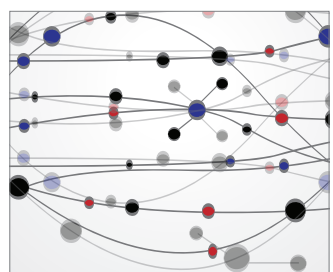

\section{The Scientific} World Journal
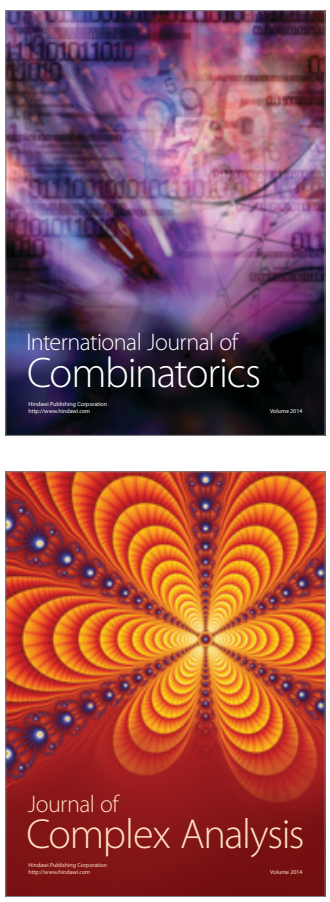

International Journal of

Mathematics and

Mathematical

Sciences
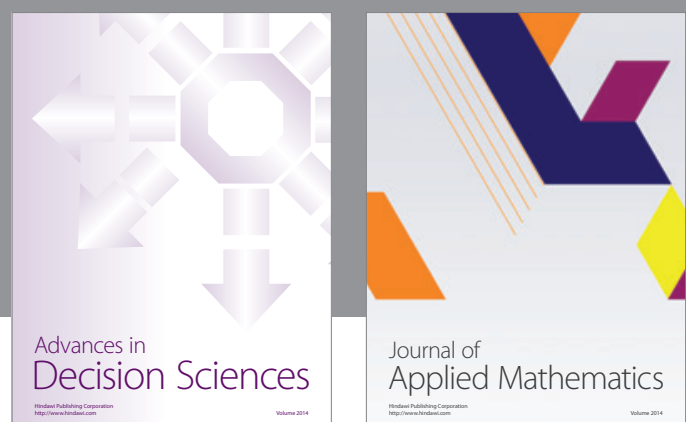

Journal of

Applied Mathematics
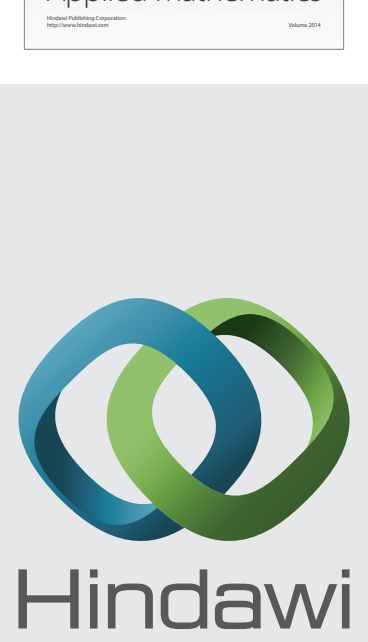

Submit your manuscripts at http://www.hindawi.com
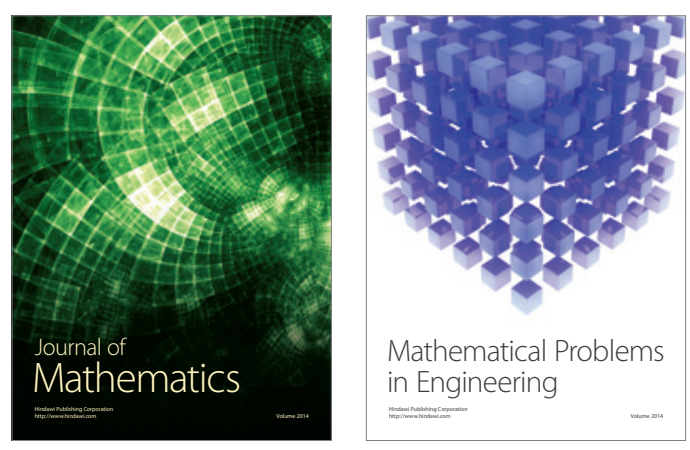

Mathematical Problems in Engineering
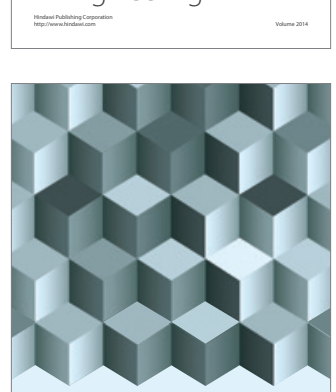

Journal of

Function Spaces
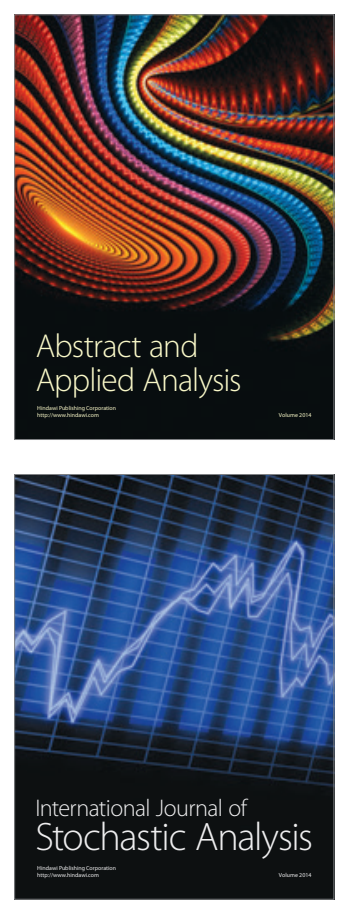

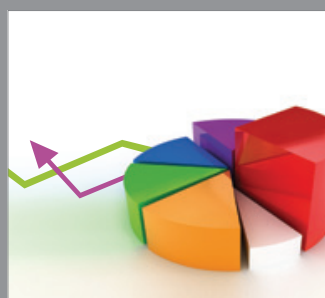

ournal of

Probability and Statistics

Promensencen
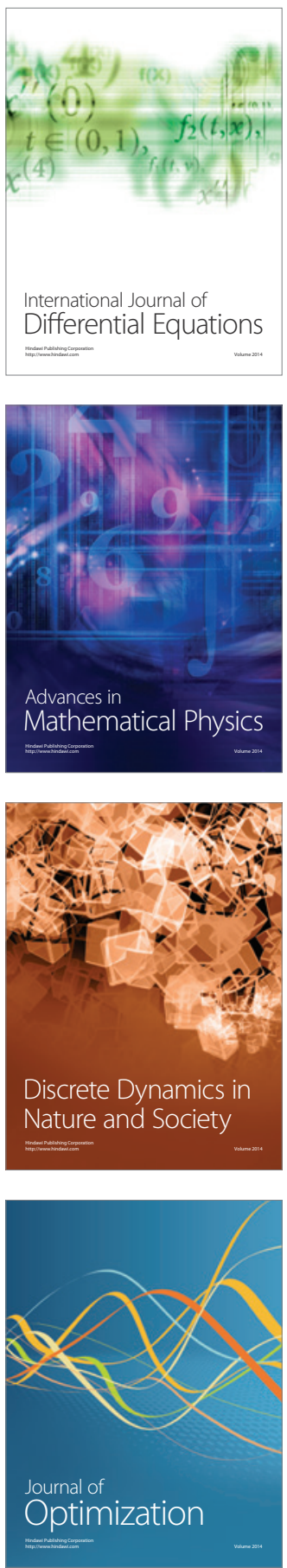